\title{
Impact of percutaneous reperfusion on ischemic mitral regurgitation in patients presented with inferior ST-segment elevation myocardial infarction
}

\begin{abstract}
Background: Ischemic mitral regurgitation (IMR) is a common complication of acute inferior ST elevation myocardial infarction (MI). Current evidences suggest that revascularization of the culprit vessels with primary percutaneous coronary artery intervention (PCI) or coronary artery bypass grafting can be beneficial for relieving IMR. The aim was to study the effect of successful primary percutaneous coronary intervention (PCI) of the culprit vessel on the degree of ischemic mitral regurgitation in patients with acute inferior STEMI.
\end{abstract}

Patients and methods: 200 patients diagnosed as acute inferior STEMI with ischemic MR \& subjected for primary percutaneous coronary intervention (PCI). Assessment of LV function and dimensions by echocardiography and assessment degree of mitral regurgitation by jet area before and after PCI.

Result: Mean MR jet area decreased from $\left(5.3 \pm 2.2 \mathrm{~cm}^{2}\right)$ to $\left(3.2 \pm 2.5 \mathrm{~cm}^{2}\right)$, (p value $\left.<0.05\right)$ after PCI, and this improvement in MR was evident in all degrees of MR. There was a significant improvement in the degree of MR among non-diabetic patients in comparison with diabetic patients. Shorter onset-to-reperfusion time and no total occlusion before $\mathrm{PCI}$ were the independent predictors of early improvement of IMR.

Conclusion: PPCI in Patients with ischemic mitral regurgitation associated with inferior STEMI led to a decrease in the severity of mitral regurgitation at 40days post PCI. No further improvement in the severity of mitral regurgitation occurred after 40days post PCI. Patients with moderate and severe mitral incompetence after 40days had a higher incidence of hospitalization with decompensated heart failure and more left ventricular remodeling changes at 6 months follow up.

Keywords: ischemic mitral incompetence, inferior STEMI, primary PCI, acute coronary syndrome, transient ischemic attack
Volume I4 Issue I - 202I

\section{Mahmoud A Abouomar, Osama Shouib, Timoor M Hassan, Ayman Elsheikh}

Department of cardiology Tanta University, Egypt

Correspondence: Mahmoud A Abouomar, Lecturer of Cardiology, Tanta University, Elgharbia governorate, Elmehala Elkobra, Egypt,Tel +2 01096085665 ,

Email maabouomar@gmail.com

Received: December 17, 2020 | Published: February 28, 202
Abbreviations: ACS, acute coronary syndrome; AMI, acute myocardial infarction; $\mathrm{CAD}$, coronary artery disease; $\mathrm{CBC}$, complete blood count; CK-MB, creatine kinase-myocardial band; DES, drug eluting stent; DM, diabetes mellitus; $\mathrm{EF}$, ejection fraction; $\mathrm{FH}$, family history; LCX, left circumflex; LV, left ventricle; LVED, left ventricle end diastolic; LVES, left ventricle end systolic; IMR, ischemic mitral regurgitation; MACEs, major adverse cardiac and cerebral events; MR, mitral regurgitation; PCI, percutaneous coronary artery intervention; RCA, right coronary artery; STEMI, ST elevation myocardial infarction; TIA, transient ischemic attack; TO, total occlusion

\section{Introduction}

Ischemic mitral regurgitation (IMR) has been recognized as an important complication after acute myocardial infarction (AMI) and is known to worsen patients prognoses even if the degree of mitral regurgitation (MR) is mild to moderate. ${ }^{1-4}$ However, the majority of previously published studies have been based on the severity of IMR in the subacute or chronic time period of myocardial infarction, with heterogeneous study populations and various reperfusion techniques.
Many factors contribute to the occurrence of IMR, including postinfarct left ventricular remodeling, papillary muscle displacement (to cause imbalanced drag and full forces of the mitral valve), contractile dysfunction of the left ventricle, structural changes of the valvular ring, and ventricular electro-mechanical dys-synchrony ${ }^{5,6}$ Of these factors, left ventricular remodeling is thought to be the most important mechanism responsible for IMR..$^{7,8}$ The entire or local remodeling of the left ventricle results in luminal distortion and enlargement, which causes displacement of the papillary muscle attachment to pull the mitral valve towards the cardiac apex. The reduced contractility of the left ventricle following AMI further contribute to the incompetence of the mitral valve, leading thus to IMR. The location of myocardial infarct also affects the probability and severity of IMR: an infarction in the inferior wall of the in the left ventricle, which causes the posterior papillary muscles to move non-symmetrically, is more likely to cause IMR than an infarction in the anterior wall.

Current interventions of IMR include conservative therapy with medications, open surgery (mitral valve replacement or repair), and percutaneous interventional therapies (percutaneous mitral annuloplasty and percutaneous edge-to-edge mitral valve repair). PCI 
and $\mathrm{CABG}$ have been shown to modify the incidence and improve the prognosis of IMR following AMI.

\section{Patients and methods}

\section{Study population}

This study was conducted in the cardiology department, Tanta University, Egypt, from June 2018 to August 2020. This study included 200 consecutive patients admitted with Inferior STEMI. The local research ethics committee approved the study in May 2018 and consent for participation of the study was taken from each patient.

Diagnosis of inferior STEMI was defined based on the presence of typical anginal pain more than 20minutes, associated with new ST-elevation at the J-point in inferior leads with the cut-point: $>1 \mathrm{~mm}$ in all inferior leas. The diagnosis was confirmed by the elevation in troponin levels. ${ }^{10}$ At baseline, all patients subjected to a detailed history, complete physical examination, routine laboratory workup $(\mathrm{CBC}$, serum urea, serum creatinine, random blood sugar, Cardiac Enzymes, liver function, and coagulation profile), Electrocardiogram at first medical contact.

\section{Echocardiographic evaluation}

At baseline, transthoracic Echocardiography is taken just before PCI; all images are taken and analyzed offline by two echocardiographers to reduce inter-observer variability, we used a GE Vivid 7 echocardiograph with $2.5 \mathrm{MHz}$ transducer. We evaluated the following; LV ejection fraction (EF); was evaluated (using the biplane method of discs. Also LV end diastolic (LVED) and LV end systolic (LVES) dimensions were measured11. The severity of ischemic MR by Jet area; The degree of mitral regurgitation was classified according to jet area into: 12 Mild MR if the Jet area $<4 \mathrm{~cm} 2$. Moderate MR if Jet area from $4-8 \mathrm{~cm}^{2}$. Severe MR if Jet area $>8 \mathrm{~cm}^{2}$.

Exclusion criteria: Patients with previous myocardial infarction, patients with wall motion abnormality in another territory not related to the inferior STEMI, patients with MR due to mechanical complication in the valve, patients with MR due to organic valvular etiology (e.g., rheumatic MR and MR due to mitral valve prolapse), presence of other mechanical complications, patients refusal to sign the consent, failure of achieving a final TIMI flow III post PCI.

\section{Angiographic procedure}

Coronary angiography and percutaneous coronary intervention were done through the femoral or radial approach, according to the choice of attending physician. All patients received the following medications: (1) A loading dose of 300mg Aspirin orally followed by $75-100 \mathrm{mg} /$ day. (2) A loading dose of $180 \mathrm{mg}$ Ticagrelor orally followed by a maintenance dose of $90 \mathrm{mg}$ twice daily or Clopidogrel $600 \mathrm{mg}$ orally followed by a maintenance dose of $75 \mathrm{mg}$ /day if Ticagrelor is contraindicated. (3) Unfractionated heparin at a dose of (100IU/ $\mathrm{kg})$, the dose was reduced to $(70 \mathrm{IU} / \mathrm{kg})$ in case of administration of glycoprotein IIb/IIIa inhibitor (eptifibatide) was given during the procedure. Primary PCI was done to all patients using DES. Successful PCI is defined as achieving TIMI III flow at the end of procedure. TIMI Grade Flow is a scoring system from 0-3 referring to levels of coronary blood after PCI. ${ }^{13}$

After the procedure, patients were transferred to the coronary care unit, and medication and follow-up of patients were left to the attending physician according to the patients' clinical condition.

\section{Follow up visits}

We scheduled two follow-up visits after 40days and 6 months from PPCI, during which all patients were subjected to a full clinical examination and follow-up echocardiography, with reevaluation of the IMR and LV function, dimensions. IMR improvement defined as improvement one degree or more. Acquired images were analyzed offline by two echocardiographers not aware of the previous echocardiographic or the clinical data of the patients. We evaluated the same parameter of the baseline procedure. We recorded any MACEs (death, hospitalization for heart failure, ACS, stroke, or TIA).

\section{Statistical analysis}

Data were collected, revised and tabulated using a standard personal computer (PC). The data were then analyzed statistically using the SPSS statistical package version. ${ }^{14}$ The following tests were done: Number. Mean \pm SD absolute numbers Percentage. Comparisons between means using $\mathrm{T}$ test of independent samples Differences between proportions were compared with the chi-square test. A value of $\mathrm{P} \leq 0.05$ was statistically significant.

\section{Results}

This study included 200 patients admitted to cardiology department at Tanta university hospital, Egypt, from June 2018 to August 2020, diagnosed as inferior STEMI and associated with ischemic mitral regurgitation, treated with primary PCI. Baseline demography and risk factors are summarized in Table 1. Ischemic MR severity before PCI; shown in Table 2. Left ventricular function and dimensions before PCI were shown in Table 3. The LV Ejection fraction was estimated by M-Mode. The mean \pm SD LVEF, LVEDD, LVESD at baseline was $49.13 \pm 10.22 \%, 54.46 \pm .6 .72$ and $38.9 \pm 7.19$ respectively.

Table I Baseline demographic and clinical characteristics of patients before $\mathrm{PPCl}$

\begin{tabular}{ll}
\hline Baseline demographic\& risk factors of patients \\
\hline Characteristics & No,(\%) \\
\hline Age & $55.8 \pm 7.587$ \\
Mean & $39-72$ \\
Range & $125(62.5)$ \\
Male gender & $80(40.0)$ \\
Diabetes mellitus & $90(45.0)$ \\
Obesity & $95(47.5)$ \\
Smoking & $98(48)$ \\
hypertension & $85(42.5)$ \\
Dyslipidemia & $80(40.0)$ \\
FH of CAD & $268 \pm 220$ \\
Laboratory data. & \\
Pre-CK, U/L & $510 \pm 716$ \\
Pre-CK-MB, U/L & 5480.0 \\
Peak CK, U/L & 2600 \\
& \\
Peak CK-MB, U/L & \\
\hline
\end{tabular}




\begin{tabular}{|c|c|}
\hline \multicolumn{2}{|c|}{ Baseline demographic\& risk factors of patients } \\
\hline Characteristics & No,(\%) \\
\hline \multicolumn{2}{|c|}{ Distribution and character of coronary artery lesion } \\
\hline Culprit lesions: & $\mathrm{No}(\%)$ \\
\hline RCA & $125(62.5)$ \\
\hline LCX & $75(37.5)$ \\
\hline Multi vessel & $130(65.0)$ \\
\hline \multicolumn{2}{|l|}{ PRE PCI TIMI score } \\
\hline Total occlusion of culprit lesion(0 TIMI score) & I54(77.0) \\
\hline Non total occlusion of culprit lesion & $46(23.0)$ \\
\hline TIMI I & $25(12.5 \%)$ \\
\hline TIMI 2 & $15(7.5 \%)$ \\
\hline TIMI 3 & $6(3.0 \%)$ \\
\hline \multicolumn{2}{|l|}{ Post PCI TIMI score } \\
\hline TIMI 3 & $200(93 \%)$ \\
\hline TIMI 2 & $0(0.0 \%)$ \\
\hline
\end{tabular}

FH, family history; CAD, coronary artery disease; LCX, left circumflex; RCA, right coronary artery; LV, left ventricle; STEMI, ST, elevation myocardial infarction

Table 2 clinical characteristics of patients after PCI (degree of MR\&LV function and MACE at 40days and 6 months

Ischemic MR severity before \& after PPCI

\begin{tabular}{|c|c|c|c|c|}
\hline Parameters & \multicolumn{2}{|l|}{ No of patients(\%) pre-PPCI } & No of patients(\%) at 40 days & $\begin{array}{l}\text { No of patients(\%) at } \\
6 \text { months }\end{array}$ \\
\hline No MR & \multicolumn{2}{|l|}{$0(0.0)$} & $64(32.0)$ & $68(34.0)$ \\
\hline Mild & \multicolumn{2}{|l|}{$110(55.0)$} & $88(44.0)$ & $84(42.0)$ \\
\hline Moderate & \multicolumn{2}{|l|}{$55(27.5)$} & $38(19.0)$ & $36(18.0)$ \\
\hline sever & \multicolumn{2}{|l|}{$35(17.5)$} & $10(5.0)$ & $12(6.0)$ \\
\hline \multicolumn{5}{|c|}{ Distribution of ischemic MR before and after PPCI } \\
\hline \multirow{2}{*}{ IMR degree pre-PCI } & \multicolumn{2}{|l|}{ IMR degree 40 days post-PCI } & \multicolumn{2}{|l|}{ IMR degree 6 months post-PCI } \\
\hline & \multicolumn{2}{|l|}{ No $M R$ in $58(52.7 \%)$} & \multicolumn{2}{|l|}{ No $M R$ in $60(54.5 \%)$} \\
\hline \multirow[t]{2}{*}{ Mild(I I0(55\%) patients) } & \multicolumn{2}{|l|}{ Mild in $52(47.3 \%)$} & \multicolumn{2}{|l|}{ Mild in $50(45.5 \%)$} \\
\hline & \multicolumn{2}{|l|}{ No $M R$ in $6(11.0 \%)$} & \multicolumn{2}{|l|}{ No $M R$ in $8(14.5 \%)$} \\
\hline \multirow[t]{3}{*}{ Moderate $(55,27.5 \%)$ patients } & \multicolumn{2}{|l|}{ Mild in $32(58.2 \%)$} & \multicolumn{2}{|l|}{ Mild in 30(54.5\%) } \\
\hline & \multicolumn{2}{|l|}{ Moderate in $17(30.8)$} & \multicolumn{2}{|l|}{ Moderate in $17(31.0)$} \\
\hline & \multicolumn{2}{|l|}{ Mild in $4(11.4 \%)$} & \multicolumn{2}{|l|}{ Mild in $4(11.4 \%)$} \\
\hline \multirow[t]{2}{*}{ Severe(35(I7.5\%) patients) } & \multicolumn{2}{|l|}{ Moderate in $2 \mathrm{I}(60.0 \%)$} & \multicolumn{2}{|l|}{ Moderate in $19(54.2 \%)$} \\
\hline & \multicolumn{2}{|l|}{ Severe in $10(28.6 \%)$} & \multicolumn{2}{|l|}{ Severe in $12(34.2 \%)$} \\
\hline \multicolumn{5}{|c|}{ MACE at flow up } \\
\hline \multirow[t]{5}{*}{ Parameter } & MR degree & At 40 days & At 6 months & \\
\hline & No MR & 0 & $\mathrm{I}(\mathrm{I} .40 \%)$ & \\
\hline & Mild MR & $1(1.13 \%)$ & $\mathrm{I}(\mathrm{I} .20 \%)$ & \\
\hline & Moderate MR & $3(7.9 \%)$ & $5(13.8 \%)$ & \\
\hline & Severe MR & $4(40 \%)$ & $6(50 \%)$ & \\
\hline Total MACE & & $8(4.0 \%)$ & $13(6.5 \%)$ & \\
\hline
\end{tabular}

MR, mitral regurge; $\mathrm{PPCl}$, primary percutaneous coronary artery interventions; $\mathrm{MACE}$, major adverse cardiac and cerebral events

Citation: Abouomar MA, Shouib O, Hassan TM, et al. Impact of percutaneous reperfusion on ischemic mitral regurgitation in patients presented with inferior ST-segment elevation myocardial infarction. / Cardiol Curr Res. 2021;14(I):17-22. DOI: 10.15406/jccr.202I.14.00502 
Table 3 Ischemic MR\&LV dimensions and function before and after $\mathrm{PCl}$

\begin{tabular}{|c|c|c|c|c|c|}
\hline \multicolumn{6}{|c|}{ Ischemic MR severity by mean of jet area before and after $\mathrm{PCI}$} \\
\hline IMR & $\begin{array}{l}\text { Before }\left(\text { jet area } \mathrm{cm}^{2}\right) \\
\text { Mean } \pm \mathrm{SD}\end{array}$ & $\begin{array}{l}\text { After (40days) (jet area } \mathrm{cm}^{2} \text { ) } \\
\text { Mean } \pm \mathrm{SD}\end{array}$ & $\begin{array}{l}\text { After } 6 \text { months (jet area } \\
\mathrm{cm}^{2} \text { ) }\end{array}$ & PValue & $\mathrm{P}^{*}$ Value \\
\hline Mild & $2.84 \pm 1.30$ & $2.10 \pm 1.454$ & $2.04 \pm 1.58$ & 0.002 & 0.79 \\
\hline Moderate & $5.933 \pm 1.74$ & $5.15 \pm 1.133$ & $5.06 \pm 1.24$ & 0.017 & 0.74 \\
\hline Sever & $6.860 \pm 1.62$ & $5.61 \pm 1.60$ & $5.62 \pm 1.50$ & 0.033 & 0.98 \\
\hline TO culprit $V$ & $5.20 \pm 2.230$ & $3.28+2.56$ & $3.40+2.48$ & $<0.0001$ & 0.69 \\
\hline DM patients & $4.70 \pm 1.95$ & $4.6 I+I .86$ & $4.68+1.64$ & 0.76 & 0.80 \\
\hline Non DM patients & $4.8 \mathrm{I} \pm 2.36$ & $3.20 \pm 2.40$ & $3.32 \pm 2.52$ & $<0.0001$ & 0.70 \\
\hline \multicolumn{6}{|c|}{ LV dimensions \& functions before \& after $\mathrm{PCI}$} \\
\hline Parameter & $\begin{array}{l}\text { Before } \mathrm{PCl} \\
\text { (mean } \pm \mathrm{SD} \text { ) }\end{array}$ & $\begin{array}{l}40 \text { days After } \mathrm{PCl},(\text { mean } \\
\pm \mathrm{SD})\end{array}$ & 6 months After $\mathrm{PCl}$ & $P$ value & $\mathrm{P}^{*}$ value \\
\hline LVEF \% & $49.13 \pm 10.22$ & $51.82 \pm 10.26$ & $52.64 \pm 10.10$ & 0.008 & 0.42 \\
\hline $\operatorname{LVEDD}(\mathrm{mm})$ & $54.46 \pm .6 .72$ & $52.76 \pm 6.79$ & $52.82 \pm 6.65$ & 0.012 & 0.92 \\
\hline $\operatorname{LVESD}(\mathrm{mm})$ & $38.9 \pm 7.19$ & $37.90 \pm 7.140$ & $36.98 \pm 7.20$ & 0.018 & 0.20 \\
\hline
\end{tabular}

CA, coronary artery; DM, diabetes mellitus; SD, standard deviations; TO, total occlusion

$\mathrm{P}$ value comparison between pre $\mathrm{PCl}$ and 40 days after $\mathrm{PCl}$. $\mathrm{P}^{*}$ value comparison between 40 days and $6 \mathrm{months}$ after $\mathrm{PPCl}$

Coronary angiography, characteristic and distribution of coronary lesion were shown in Table 1. All patients were subjected to primary percutaneous coronary intervention (PCI) of culprit lesions only. All patients had successful PCI with TIM III flow.

Ischemic MR severity 40days after PCI, at the first flow up visit after 40days, IMR was improved in 121 patients $(60.5 \%)$, in 64 patients $(32.2 \%)$ the IMR was completely disappeared, 88 patients (44.0\%) had mild MR, 38 patients (19\%) had moderate MR while 5 patients (10\%) still had severe MR at 40days post PCI, (Table 2). Flow up visits at 6months; at six months all patients flowed clinically and by echocardiography, no significant changes in degree of IMR in comparison to what were at the 40days flow up (Table 3), the patient with moderate and severe IMR have more MACE than that with mild IMR, where 6 patients from 12 patients with severe IMR have MACE mostly hospitalization for heart failure.

Effect of PCI on different degree of ischemic MR: Mean MR jet area decreased from $\left(5.3 \pm 2.2 \mathrm{~cm}^{2}\right)$ to $(3.2 \pm 2.5 \mathrm{~cm} 2)$, ( $\mathrm{p}$ value $\left.<0.05\right)$ after PCI, and this improvement in MR was evident in all degrees of MR as shown in Table 2. After 6months there were no significant changes in degree of IMR in comparison to that in 40days flow up visits (Table 2).

Effect of PCI on LV dimensions \& function: There was an evident improvement in LV dimensions \& function as shown in Table 2. Where LVEF, LVED and LVES dimensions improved significantly at 40 days flow up, whoever at 6 months flow up no more improve in echocardiographic parameter in patients get improved at 40days , patients with severe mitral incompetence at 40days after PPCI get more worse at 6 months flow up and the MACEs occur in more than half of those patients mostly in form of hospitalization for heart failure (Table 2), regarding echocardiographic dimensions in patients with severe mitral incompetence at 40days, the LVEDD and LVESD get more dilatation at 6month follow up, on the other hand patients with mild and moderate mitral incompetence no significant changes in LV dimensions and LFEF.
Predictors of IMR improvement; Multivariable analysis showed that shorter onset-to-reperfusion time and TIMI $\geq 1$ at initial angiography were the independent predictors of improvement in IMR early after primary PCI. Post-PCI TIMI grade (2 or 3 ) did not have a significant impact on the improvement of IMR after PCI. On the contrary, lower peak CK-MB level was an independent predictor of improvement in IMR after primary PCI (early post PCI to late postPCI). Higher age, lower EF at the acute phase, was the independent predictors of persistent $\geq$ mild IMR at 40days and 6months after primary PCI (Table 4).

Table 4 Factors predicting IMR changes

\begin{tabular}{llll}
\hline Parameters & \multicolumn{1}{l}{ Odds Ratio } & $\mathbf{9 5 \%} \mathbf{C l}$ & P Value \\
\hline Onset-to-reperfusion time, h & 0.94 & $0.91 \mathrm{I}-0.980$ & 0.02 \\
Non total occlusion & 2.202 & $1.205-4.160$ & 0.01 \\
Peak CK-MB, U/L & 0.984 & $0.980-1.100$ & 0.03 \\
Ejection fraction before PCl, \% & 0.941 & $0.938-0.962$ & 0.003 \\
Age & 1.018 & $1.002-1.082$ & $<0.001$ \\
\hline
\end{tabular}

$\mathrm{Cl}$ indicates confidence interval; CK-MB, creatine kinase-myocardial band; $I M R$, ischemic mitral regurgitation; $\mathrm{PCl}$, percutaneous coronary intervention

\section{Discussion}

It is estimated that $1 / 5$ of the IMR cases were due to myocardial infarction, and half of the IMR cases are complicated by congestive heart failure. The incidence of IMR is $40 \%$ following AMI and a 5-year mortality rate up to $62 \% .{ }^{15-17}$ Presence of ischemic MR carries an adverse prognosis in acute STEMI patients. Patients at risk are elder, more likely to be female, to have lower EF and multi-vessel $\mathrm{CAD}$, these findings are well acknowledged in previous reports. ${ }^{18-20}$

Many factors contribute to the occurrence of IMR, including postinfarct LV remodeling, papillary muscle displacement, contractile dysfunction of the left ventricle, structural changes of the valvular 
ring, and ventricular electro-mechanical dys-synchrony. 5,6 Of these factors, left ventricular remodeling is thought to be the most important mechanism responsible for IMR. ${ }^{7,8}$ On this basis, coronary blood flow restoration reduces LV remodeling and improves LV function, this strategy should attenuate ischemic mitral regurgitation. ${ }^{21}$

In the present study, we retrospectively examined the dynamics of IMR after primary PCI for acute inferior MI, to clarify (1) the acute effect of primary PCI on IMR and its relation to the degree of acute changes in LV parameters and (2) the chronic effect of primary PCI on IMR along with chronic LV remodeling/reverse remodeling. We also evaluated the predictors of changes in the IMR and the resulting prognostic impact of IMR in each clinical stage after primary PCI.

The degrees of IMR also changed in the chronic stage, reflecting the degree of LV remodeling/reverse remodeling in the chronic phase after AMI. Age, peak CK-MB and pre-EF were independent predictors of the existence of IMR in the follow-up phase. Existence of clinically significant IMR in the acute and chronic phases of AMI significantly worsened the short-term and long-term prognosis after PCI, respectively. We evaluated 200 patients with mild, moderate or severe ischemic MR as confirmed by echocardiography, who underwent primary PCI after inferior STEMI. They were followed 40days and six months after PCI by echocardiography.

The principle finding of the present study was a significant improvement in ischemic MR degree after primary PCI: in all degree of IMR, Table 2, at 6months follow up no significant more improve in IMR degree.

Our results came in concordance with Sarah C. and colleagues ${ }^{22}$ concluded that the PCI is more effective in decreasing the incidence of moderate or severe IMR for myocardial infarction, compared to results for patients with no PCI. Fayaz A et al, ${ }^{23}$ demonstrated the efficacy of angioplasty in the treatment of severe mitral regurgitation secondary to ischemic posterior papillary muscle dysfunction.

All patients in our study were treated with primary PCI for culprit lesion only, and after 2 weeks the patients subjected to complete revascularization. Conventional management strategies for treating STEMI patients with multi-vessel CAD may vary from complete interventional revascularization including non-culprit lesions during index procedure to medical therapy, staged PCI or subsequent surgical revascularization. ${ }^{24-26}$ Impact of completeness of revascularization and residual ischemic myocardial burden on ischemic MR is a potential direction for future investigations. IMR could newly develop or worsen even though the culprit lesion was successfully revascularized. Acute changes in the magnitude of IMR were related to the degree of LV changes, which would reflect the amount of rescued myocardium by emergency revascularization. The earlier the onset-to-reperfusion time, the greater the degree of rapid LV improvement is expected.

Time to reperfusion is an important factor in salvaging the myocardium at risk. Early reperfusion improves immediate and long-term outcomes in patients who present with STEMI. ${ }^{27,28}$ We have also found that longer ischemic time prior to PCI is an important determinant in the severity of IMR. This finding once again emphasizes the notion of early opening of the infarcted-artery with the aim of salvaging as much muscle mass as possible; therefore reducing the rate of IMR occurrence.

The presence of moderate or severe IMR, multivessel disease or an LVESD at baseline $\geq 40 \mathrm{~mm}$ predict patients worse late clinical outcomes. Importantly, patients with moderate to severe IMR experience a higher incidence of six months MACE, which is mainly driven by higher rates of hospitalization for heart failure. Functional IMR has an important prognostic impact after myocardial infarction. Previous studies have shown IMR to be an independent predictor of outcome as it doubled mortality rates and increased the incidence of hospital admissions for heart failure. ${ }^{29-31}$

In the current study, there was a significant difference $(\mathrm{P}<0.001)$ between diabetic and non-diabetic patients with better outcome regarding the improvement in the severity of IMR among non-diabetic patients. This could be explained by the fact that diabetic vessel may have more extensive atherosclerosis and microcirculation could be more damaged. This was in agreement with work of Verghese Mathew and colleague, ${ }^{32}$ who reported that outcomes in patients with diabetes mellitus undergoing percutaneous coronary intervention in 9month follow up is associated with significant adverse events $(\mathrm{P}$ value $<0.05)$ including significant mitral regurgitation.

Ischemic MR in multivessel vs. single vessel disease; in the present study, 130 patients $(65.0 \%)$ with acute inferior STEMI and ischemic MR had multivessel disease while 70 patients $(35.0 \%)$ had single vessel. This is similar to what observed by Pellizzon G, et $\mathrm{al},{ }^{33}$ that found the presence of ischemic MR is associated with triple-vessel coronary artery disease. Anselmi M. and colleagues 34 also demonstrated that patients with MR had more severe coronary disease, with a greater incidence of three vessel disease and a lower incidence of single vessel disease than did patients without MR.

\section{Limitations}

Echocardiographic data is observed after the MI and do not exclude that some MR was present beforehand in some patients. Nonetheless, our prevalence exceeds what has been documented in the general population therefore; it is most likely attributed to the MI. Second, the present study had a limited study population, which was restricted to patients who fulfilled predefined inclusion criteria. Therefore, our results cannot be directly deduced to other subgroups of patients, such as those not receiving emergency PCI. Fourth, the impact of IMR on the outcome of AMI may have been influenced by the concomitant treatment with $\beta$-blockers and angiotensinconverting enzyme inhibitors. Although we could not include those medications as possible variables for the outcome in the present study, such medications should be accounted for in the analysis of outcome.

\section{Conclusion}

PPCI in Patients with ischemic mitral regurgitation associated with inferior STEMI led to a decrease in the severity of mitral regurgitation at 40 days post PCI. No further significant improvement in the severity of mitral regurgitation occurred after 40 days post PCI. Patients with moderate and severe mitral incompetence after 40days had a higher incidence of hospitalization with decompensated heart failure and more left ventricular remodeling changes at 6 months follow up. The existence of acute IMR on arrival and persistent IMR in the chronic phase after primary PCI worsened the short-term and long-term prognosis of AMI, respectively. Early reperfusion contributed to the improvement of IMR after PCI. Earlier revascularization is thought to be a key to prevent persistent IMR and thus improve the long-term prognosis of AMI.

\section{Acknowledgments}

We would like to thank our colleagues, nurses, and technicians in the cath lab in the cardiology department for help in collecting data and completion of our study 


\section{Conflicts of interest}

The authors declare that they have no conflicts of interest concerning this paper.

\section{Funding}

None.

\section{References}

1. Feinberg MS, Schwammenthal E, Shlizerman L, et al. Prognostic significance of mild mitral regurgitation by color Doppler echocardiography in acute myocardial infarction. Am J Cardiol. 2000;86(9):903-907.

2. Grigioni F, Enriquez-Sarano M, Zehr KJ, et al. Ischemic mitral regurgitation:long-term outcome and prognostic implications with quantitative Doppler assessment. Circulation. 2001;103(13):1759-1764.

3. Bursi F, Enriquez-Sarano M, Nkomo VT, et al. Heart failure and death after myocardial infarction in the community:the emerging role of mitral regurgitation. Circulation. 2005;111(3):295-301.

4. Bursi F, Enriquez-Sarano M, Jacobsen SJ, et al. Mitral regurgitation after myocardial infarction:a review. Am J Med. 2006;119(2):103-112.

5. Ge L, Wu Y, Soleimani M, et al. Moderate ischemic mitral regurgitation after posterolateral myocardial infarction in sheep alters left ventricular shear but not normal strain in the infarct and infarct borderzone. Ann Thorac Surg. 2016;101(5):1691-1699.

6. Levine RA, Hagége AA, Judge DP, et al. Mitral valve diseasemorphology and mechanisms. Nat Rev Cardiol. 2015;12(12):689-710.

7. Szymanski C, Bel A, Cohen I, Touchot B, et al. Comprehensive annular and subvalvular repair of chronic ischemic mitral regurgitation improves long-term results with the least ventricular remodeling. Circulation. 2012;126(23):2720-2727.

8. Soleimani M, Khazalpour M, Cheng G, et al. Moderate mitral regurgitation accelerates left ventricular remodeling after posterolateral myocardial infarction. Ann Thorac Surg. 2011;92(5):1614-1620.

9. Huang WL, Fu Q. Development in mechanisms of ischemic mitral regurgitation. Chin Med J (Engl). 2013;126(90:1766-1770.

10. Thygesen K, Alpert JS, Jaffe AS, et al. Fourth Universal Definition of Myocardial Infarction (2018). J Am Coll Cardiol. 2018;72(18):22312264.

11. Lang RM, Badano LP, Mor-Avi V, et al. Recommendations for cardiac chamber quantification by echocardiography in adults:An update from the American Society of Echocardiography and the European Association of Cardiovascular Imaging. J Am Soc Echocardiogr. 2015;28(1):1-39.e14.

12. Luthra A. Assessment of MR severity by colour flow mapping. Echo Made Easy. 2005;81:8061-436.

13. TIMI Study Group. The Thrombolysis in Myocardial Infarction (TIMI) trial, Phase I findings. N Engl J Med. 1985;312(14):932-936.

14. Kirkpatrick LA, Feeney BC. A simple guide to IBM SPSS statistics for version 20.0. USA: Cengage Learning; 2013.

15. Aronson D, Goldsher N, Zukermann R, et al. Ishemic mitral regurgitation and risk of heart failure after myocardial infarction. Arch Intern Med. 2006;166(21):2362-2368.

16. Huang WL, Fu Q. Development in mechanisms of ischemic mitral regurgitation. Chin Med J (Engl). 2013;126(9):1766-1770.

17. Chau S, Hung J, Chung SY, et al. Primary percutaneous coronary intervention lowers the incidence of ischemic mitral regurgitation in patients with acute ST-elevated myocardial infarction. Circ J. 2010;74(11):2386-2392.
18. López-Pérez M, Estévez-Loureiro R, López-Sainz A, et al. Long-term prognostic value of mitral regurgitation in patients with ST-segment elevation myocardial infarction treated by primary percutaneous coronary intervention. Am J Cardiol. 2014;113(6):907-912.

19. Pellizzon GG, Grines CL, Cox DA, et al. Importance of mitral regurgitation in patients undergoing percutaneous coronary intervention for acute myocardial infarction:the controlled abciximab and device investigation to lower late angioplasty complications (CADILLAC) trial. J Am Coll Cardiol. 2004;43(8):1368-1374.

20. Valuckiene Z, Urbonaite D, Jurkevicius R. Functional (ischemic) mitral regurgitation in acute phase of myocardial infarction:associated clinical factors and in-hospital outcomes. Medicina (Kaunas). 2015;51(2):92-99.

21. Trichon BH, Glower DD, Shaw LK, et al. Survival after coronary revascularization, with and without mitral valve surgery in patients with ischemic mitral regurgitation. Circulation. 2003;108(Suppl 1):103-110.

22. Sarah C, Judy H, Sheng-Ying C, et al. Primary Percutaneous Coronary Intervention Lowers the Incidence of Ischemic Mitral Regurgitation in Patients With Acute ST-Elevated Myocardial Infarction. Circ J. 2010;74(11):2386-2392.

23. Fayaz A, Shawl, Mervyn B, et al. Emergent Coronary Angioplasty in the Treatment of Acute Ischemic Mitral Regurgitation:Long- Term Results in Five Cases. J Am Co11 Cardiol. 1989;14(4):986-991.

24. Wald DS, Morris JK, Wald NJ, et al. Randomized trial of preventive angioplasty in myocardial infarction. $N$ Engl J Med. 2013;369(12):11151123 .

25. Engstrøm T, Kelbæk H, Helqvist S, et al. Complete revascularisation versus treatment of the culprit lesion only in patients with ST-segment elevation myocardial infarction and multivessel disease (DANAMI-3PRIMULTI): an open-label, randomised controlled trial. Lancet. 2015;386(9994):665-671.

26. Weitemeyer REM, Murphy SP, Gillen R, et al. Management and outcomes of significant non-culprit coronary artery lesions in STEMI:a retrospective cohort study Int. Cardiovasc Forum J. 2015;3(2015):14-19.

27. Chesebro JH, Knatterud G, Roberts R, et al. Thrombolysis in myocardial infarction (TIMI) trial, phase I: a comparison between intravenous tissue plasminogen activator and intravenous streptokinase. Clinical findings through hospital discharge. Circulation. 1987;76(1):142-154.

28. Armstrong PW, Gershlick AH, Goldstein P, et al. Fibrinolysis or primary PCI in ST-segment elevation myocardial infarction. $N$ Engl $\mathrm{J}$ Med. 2013;368(15):1379-1387.

29. Amigoni M, Meris A, Thune JJ, et al. Mitral regurgitation in myocardial infarction complicated by heart failure, left ventricular dysfunction, or both:prognostic significance and relation to ventricular size and function. Eur Heart J. 2007;28(3)326-333.

30. Grigioni F, Enriquez-Sarano M, Zehr KJ, et al. Ischemic mitral regurgitation:long-term outcome and prognostic implications with quantitative doppler assessment. Circulation. 2001;103(13):1759-1764.

31. Lamas GA, Mitchell GF, Flaker GC, et al. Clinical significance of mitral regurgitation after acute myocardial infarction. Survival and ventricular enlargement investigators. Circulation. 1997;96(3):827-833.

32. Prasad A, Mathew V, Holmes DR, et al. Current management of nonSTsegment- elevation acute coronary syndrome: reconciling the results of randomized controlled trials. Eur Heart J. 2003;24(17):1544-1553.

33. Pellizzon G, Grines C, Cox D. Importance of mitral regurgitation in patients undergoing percutaneous coronary intervention for acute myocardial infarction. J Am Coll Cardiol. 2004;43(8):1368-1374.

34. Anselmi M, Masud M. Significant mitral regurgitation in a first inferior myocardial infarction. J Am Coll Cardiol. 1998;20:158-193. 